3

4 Dr Lihai Zhang (Corresponding author)

5 Department of Infrastructure Engineering

6 The University of Melbourne, Victoria 3010, Australia

7 Email: lihzhang@unimelb.edu.au

Dr Saeed Miramini

Department of Infrastructure Engineering

The University of Melbourne, Victoria 3010, Australia

Email: s.miramini@unimelb.edu.au

Associate Professor Martin Richardson 3121, Australia

Email: orthovic@gmail.com

Professor Priyan Mendis

Department of Infrastructure Engineering

The University of Melbourne, Victoria 3010, Australia

Email: pamendis@unimelb.edu.au

Professor Peter Ebeling

Email: peter.ebeling@monash.edu

\title{
The role of impairment of mesenchymal stem cell function in osteoporotic bone fracture healing
}

Department of Surgery, University of Melbourne, Epworth Hospital, Richmond, Victoria

Department of Medicine, Monash University, Clayton VIC 3168, Australia 
29 With demographic change and increasing life expectancy, osteoporotic fractures have become one of the most prevalent trauma conditions seen in daily clinical practice. A variety of factors are known to affect the rate of healing in osteoporotic conditions (e.g. both biochemical and biomechanical environment of callus cells). However, the influence of impairment of mesenchymal stem cell function in the osteoporotic condition on bone fracture healing has not been fully understood. In the present study, we develop a mathematical model that quantifies the change in biological processes within the fracture callus as a result of osteoporosis. The model includes special features of osteoporosis such as reduction in mesenchymal stem cell (MSC) number in osteoporotic bone, impaired response of osteoporotic MSCs to their biomechanical microenvironment and the effects of configuration of locking compression plate (LCP) system on healing in this context. The results presented here suggest that mechanical-mediated MSC differentiation at early stages of healing are significantly affected under osteoporotic conditions, while it is predicted that the flexible conditions.

Key words: Mesenchymal stem cells; osteoporosis; bone fracture healing; mechanoregulation; locking compression plate. 
52 As the world's population is ageing rapidly, osteoporosis becomes a highly prevalent and costly disease which is predicted to affect 1,555 million worldwide by 2050 [1]. The most frequent fractures among elderly patients are associated with osteoporosis [2]. Compared with normal fracture healing, osteoporotic fracture healing of the femoral diaphysis is

significantly delayed by around three weeks [3]. In addition, biomechanical evaluation of osteoporotic healing using a sheep model also demonstrated a delay of fracture healing in osteoporotic bones with a significant reduction in torsional and bending stiffness by $33 \%$ and $21 \%$, respectively, in comparison to normal healing[4].

The ability of biological tissues to repair themselves following trauma depends on the biological actions of mesenchymal stem cells (MSCs), which are gradually diminished with increasing age [5]. Immediately after a fracture, MSCs migrate into the fracture site, where they exert their biological actions by responding to their biochemical and biomechanical microenvironment [6]. However, clinical studies have shown that the repair capacity of ageing bone progressively declines as a result of a deficit of bone MSCs [7]. Further, MSCs from osteoporotic patients feature impaired signal transduction which could have a negative impact on callus formation, endochondral ossification and bone morphogenetic protein (BMP) mediated MSC migration and invasion, ultimately a delayed healing [8,9].

It is well known that mechano-regulation plays an important role in the course of bone healing [10-13], especially at the early stage of healing [14]. Recent studies generally agree that the octahedral shear strain and interstitial fluid velocity are two mechanical stimuli that modulate callus cell proliferation and differentiation by triggering a cellular response [15]. However, bone cells could have an impaired response to their mechanical microenvironment [16] due to relatively fewer mesenchymal stem cells and impaired proliferative response in 
osteoporotic bone $[17,18]$.

The investigation of the effects of the stress-driven flow of interstitial fluid on bone cells showed that, after one hour of stress-driven flow of interstitial fluid, the mechanical stimuli mediated upregulation of prostaglandin $E_{2}$ and TGF- $\beta$ is significantly reduced in osteoporotic patients in comparison to normal subjects [16]. Using a series of theoretical approaches in conjunction with mechanical testing, our recent studies have suggested that the healing outcomes could be enhanced by increasing interfragmentary (IFM) movement through making locking compression plate (LCP) system more flexible by adjusting the bone-plate distance and working length of LCP [19-24]. Therefore, it is reasonable to speculate that the fixation design of LCP can be strategically modified to achieve improved fracture healing outcomes in osteoporotic bone.

Despite conclusive evidence that there is delayed and/or impaired fracture healing in osteoporotic bone, the influence of osteoporosis on fracture healing has not yet been fully understood and clinical evidence is still lacking. As shown in Fig 1.a, the purpose of this study is to develop a computational model to investigate the impaired facture healing resulting from the deficient response of MSCs in osteoporotic bone and explore the effective ways of improving the healing outcomes by strategically modifying the configuration of LCP.

\section{METHODS}

While the detailed mechanisms of age-related osteoporotic bone fracture healing are far from clear to date, we make the following assumptions for simplicity and without sacrificing the fundamental behaviours exhibited by the complicated healing processes:

- Immediately after fracture, MSCs are released into the callus tissue from the periosteum, surrounding soft tissues and bone marrow[25]. 
- The differentiation rates of MSCs into osteoblasts, chondrocytes and fibroblasts are mainly regulated by their biomechanical microenvironment of the cells [25], while the effects of growth factors (e.g. chondrogenic growth factor and osteogenic growth factor) [26] are ignored in this study.

- The impact of osteoporosis is mainly on the reduction of MSCs number in bone and impaired response of MSCs to their mechanical stimuli [16-18].

- The major source of fibroblasts, chondrocytes and osteoblasts in the callus is mainly from the differentiation of MSCs.

- The degradation and proliferation of MSCs, fibroblasts, chondrocytes and osteoblasts in callus are ignored in this present study.

Below, we present the modelling approach and formulations employed in this study.

\section{Formulations}

The methodology proposed in present study is based on the theory of porous media to model the mechanical behaviour of soft fracture callus at early stage of healing [21,22,19,23,13,27].

The callus is treated as a triphasic mixture consisting of (1) a solid phase representing extracelluar matrix (ECM) of callus; (2) a fluid phase representing interstitial fluid; and (3) a solute phase consisting of callus cells (i.e. MSCs, chondrocytes, osteoblasts and fibroblasts) which migrate within the callus. As the volume of solute phase is relatively small compared to solid and fluid phases, we may assume

$\phi^{s}+\phi^{f}=1$

where $\phi^{s}$ and $\phi^{f}$ are volume fraction of solid and fluid phase, respectively.

\section{Transport of MSCs $\left(c^{m}\right)$}

The transport of MSCs within the callus can be expressed as: 
$123 \frac{d\left(\phi^{f} c^{m}\right)}{d t}=-\nabla \bullet\left(-\phi^{f} D_{m} \nabla c^{m}+\phi^{f} \mathbf{v}^{\mathbf{f}} c^{m}\right)-\phi^{f}\left(s_{d}^{f b}+s_{d}^{c}+s_{d}^{b}\right) c^{m}-k_{d}^{m}$

where $D_{m}$ is diffusion coefficient of MSCs and $\mathbf{v}^{\mathbf{f}}$ is the true fluid velocity related to a fixed representative element volume (REV). $s_{d}^{f b}, s_{d}^{c}$ and $s_{d}^{b}$ are the differentiation rates of MSCs into fibroblasts, chondrocytes and osteoblasts, respectively. $k_{d}^{m}$ is the degradation rate of MSCs, and $\nabla \bullet$ denotes the divergence operator.

Transport of fibroblasts $\left(c^{f b}\right)$

$\frac{d\left(\phi^{f} c^{f b}\right)}{d t}=-\nabla \bullet\left(-\phi^{f} D_{f b} \nabla c^{f b}+\phi^{f} \mathbf{v}^{\mathbf{f}} c^{f b}\right)+\phi^{f} s_{d}^{f b} c^{m}-s_{d}^{f b}$

where $D_{f b}$ is diffusion coefficient of fibroblasts, and $s_{d}^{f b}$ the degradation rate of fibroblasts.

Transport of chondrocytes $\left(c^{c}\right)$

$\frac{d\left(\phi^{f} c^{c}\right)}{d t}=-\nabla \bullet\left(-\phi^{f} D_{c} \nabla c^{c}+\phi^{f} \mathbf{v}^{\mathbf{f}} c^{c}\right)+\phi^{f} s_{d}^{c} c^{m}-s_{d}^{c}$

where $D_{c}$ is diffusion coefficient of chondrocytes, and $s_{d}^{c}$ the degradation rate of chondrocytes.

Transport of osteoblasts $\left(c^{b}\right)$

$\frac{d\left(\phi^{f} c^{b}\right)}{d t}=-\nabla \bullet\left(-\phi^{f} D_{b} \nabla c^{b}+\phi^{f} \mathbf{v}^{\mathbf{f}} c^{b}\right)+\phi^{f} s_{d}^{b} c^{m}-s_{d}^{b}$

where $D_{b}$ is diffusion coefficient of osteoblasts, and $s_{d}^{c}$ the degradation rate of osteoblasts.

Modelling mechanical behaviour of soft fracture callus at early stage of healing

Traditionally, the mechanical behaviour of soft tissues such as callus is modelled using the theory of porous media with considering infinitesimal strain theory in the formulation and 
applying a linear elastic constitutive relation for modelling the solid phase. However, the soft fracture callus at early stage of healing can undergo very large deformation under physiologically relevant loading (i.e. up to $100 \%$ without rupture) [28], and therefore application of infinitesimal strain theory cannot accurately capture the mechanical behaviour of soft callus. In this study the large-deformation mechanical behaviour of early fracture callus is modelled by formulating the classical theory of porous media in Lagrangian framework.

Assuming the early stage of fracture callus is homogeneous and undergoing large deformation, the mechanical quantities (e.g. interstitial fluid velocity, $\mathbf{v}^{\mathbf{f}}$ ) can be determined through the conservation of linear momentum [29], that is

$$
\nabla \bullet\left(-p \mathbf{I}+\sigma_{E}^{s}\right)=0
$$

where $\sigma_{E}^{s}$ is effective stress of solid phase which can be modelled by using the incompressible Neo-Hookean material model which is widely used in describing the large deformation behaviour of biological tissues (e.g. soft callus) [30,29], that is

$\sigma_{E}^{s}=\frac{1}{J^{s}} \mathbf{F}^{\mathrm{s}} \cdot 2 \frac{\partial\left(\mathbf{W}^{\mathrm{s}}\right)}{\partial \mathbf{C}^{\mathrm{s}}} \cdot \mathbf{F}^{\mathbf{s}^{\mathrm{T}}}$

$$
\mathbf{W}^{\mathrm{s}}=C_{1}\left(\bar{I}_{1}-3\right)+D_{1}\left(J^{s}-1\right)^{2}
$$

$C_{1}=\mu_{0} / 2$

$D_{1}=K_{0} / 2$

where $J^{s}$ is the Jacobian determinant of the deformation gradient of the solid phase [31], $\mathbf{F}^{\mathbf{s}}$ the deformation gradient of the solid phase and $\mathbf{C}^{\mathbf{s}}$ the right Cauchy-Green deformation tensor of the solid phase. $\mathbf{W}^{\mathrm{s}}$ is the energy density function of incompressible Neo-Hookean model, $\bar{I}_{1}$ the first invariant of the isochoric part of the right Cauchy-Green deformation 
tensor, $\mu_{0}$ the initial shear modulus, and $K_{0}$ the initial bulk modulus.

The governing equation which links the calls ECM deformation to the interstitial fluid motion can be obtained through conservation of mass of solid and fluid phase with consideration of the solid reference configuration to be the "true" reference configuration and redefined all fluid quantities with respect to the solid skeleton [31], that is

$$
\dot{\mathbf{W}}+\mathbf{k} \bullet \nabla_{0} p=0
$$

$$
\dot{\mathbf{W}}=J^{s} \mathbf{F}^{\mathbf{s}^{-1}} \cdot\left[\boldsymbol{\phi}^{f}\left(\mathbf{v}^{\mathbf{f}}-\mathbf{v}^{\mathbf{s}}\right)\right]
$$

$$
\mathbf{k}=J^{s} \mathbf{F}^{\mathrm{s}^{-1}} \bullet \boldsymbol{K} \bullet \mathbf{F}^{\mathrm{s}^{-t}}
$$

where $\dot{\mathbf{W}}$ is the Lagrangian fluid velocity relative to the solid phase, $\nabla_{0}$ the material gradient operator on solid phase, and $\boldsymbol{\kappa}$ the symmetric Darcy permeability tensor.

\section{Mechano-regulation}

Experimental evidence suggest that the MSCs response to mechanical stimuli exhibits a threshold behaviour, with different differentiation behaviours triggered when the value of "mechanical stimulation index (S)" reaching different thresholds [32], i.e. (1) osteoblast differentiation when $S<1$; (2) chondrocyte differentiation when $1<S<3$; and (3) fibroblast differentiation when $S>1$. The value of mechanical stimulation index $(S)$ is determined by both octahedral shear strain of the solid phase $\left(\tau^{s}\right)$ and the interstitial fluid velocity relative to solid phase $\left(v^{f}, \mu m / s\right)[32]$, that is

$$
S=k_{d}^{s} \times\left(\frac{\tau^{s}}{a}+\frac{v^{f}}{b}\right)
$$

where $a=0.0375$ and $b=3 \mu \mathrm{m} / \mathrm{s}$ [32]. $k_{d}^{s}$ is the reduction factor of MSCs response to mechanical stimuli $\left(0 \leq k_{d}^{s} \leq 1\right)$ due to the impact of osteoporosis. 
As shown in Fig 1.b, a fractured human tibia with a $3 \mathrm{~mm}$ fracture gap size supported by a locking compression plate (LCP) was modelled in this study. It was assumed that the fracture is supported by a $4.5 \mathrm{~mm}$ broad DePuy Synthes stainless steel LCP. The plates were $206 \mathrm{~mm}$ long, $17.5 \mathrm{~mm}$ wide and $5.2 \mathrm{~mm}$ thick with 11 locking holes. The 3D geometries of tibia and LCP were created by using our previously developed techniques [22,21] involving CT scan images in conjunction with commercial medical imaging processing software Mimics (Materialise, Belgium) and CAD software Solidworks.

\section{Loading protocol}

The fractured bone was subject to a quasi-static compressive load (i.e. $150 \mathrm{~N}$ in total, equivalent to approximately $20 \%$ of the normal body weight, which is suitable for a patient after operation [21]) uniformly distributed on the top of the cortical bone.

\section{Boundary conditions}

It is assumed that the exterior boundary of early stage fracture callus is impermeable (i.e. no flux) [33]. Further, it is assumed that the majority of MSCs are from the exterior boundary of periosteal callus and bone marrow [25], that is

$\bar{c}_{0}^{m}=k_{d}^{m} c_{0}^{m}$

where $c_{0}^{m}$ is MSCs concentration at exterior boundary of periosteal callus and bone marrow under normal condition. $k_{d}^{m}$ is reduction factor $\left(0 \leq k_{d}^{m} \leq 1\right)$ due to the impact of osteoporosis.

\section{Initial conditions}

It is assumed that initially there is no cell in fracture callus, that is

$c^{m}(t=0)=0, c^{c}(t=0)=0, c^{b}(t=0)=0, c^{f b}(t=0)=0$ 


\section{Numerical considerations}

The governing equations (2)-(8) were solved numerically by using commercial finite element (FE) package COMSOL MULTIPHYSICS [34]. The soft fracture callus was meshed with 14,335 second-order tetrahedral elements (Fig 1.b), which were determined based on convergence study with an error tolerance of $2 \%$. The values of parameters used in this simulation are listed in Table 1.

The present study compared the numerical predictions of three scenarios (i.e. Cases 1-3 in Table 1) representing the impact of osteoporosis on the number of MSCs in bone $\left(k_{d}^{m}=0.5\right.$ , where $k_{d}^{m}$ is the reduction factor of MSCs number in bone as shown in Equation 10), the impaired response of MSCs to their biomechanical microenvironment $\left(k_{d}^{s}=0.5\right.$, where $k_{d}^{s}$ is the reduction factor of MSCs response to mechanical stimuli as shown in Equation 9) under different LCP configurations (i.e. bone-plate distance $=2 \sim 4 \mathrm{~mm}$ ) relative to Control (i.e. normal condition: $k_{d}^{m}=1, k_{d}^{s}=1$ and bone-plate distance $=2 \mathrm{~mm}$ ).

\section{RESULTS}

Fig 2 shows the distribution of the mechanical simulation index $(S)$ across the fracture callus (Section A-A) with consideration of the impact of osteoporosis and different boneplate distance ranging from $2 \mathrm{~mm}$ to $4 \mathrm{~mm}$. It can be seen from Fig $2 \mathrm{~b}$ (Case 1) that, compared to normal condition (Control), the mechanical simulation index $(S)$ is significantly reduced due to the impaired response of MSCs. However, with the increase of the flexibility of LCP (i.e. increasing bone-plate distance from $2 \mathrm{~mm}$ to $4 \mathrm{~mm}$ ), the impact of osteoporosis on mechanical stimuli can be alleviated as shown in Fig 2c-d.

The role of the flexibility of LCP in alleviating the impaired healing resulting from 
osteoporosis was further investigated by simulating the change in callus MSCs differentiation

to osteoblasts, chondroblasts and fibroblasts under different LCP configurations (i.e. boneplate distances) (Fig 3). It can be seen that, in comparison to Case 1 (i.e. bone-plate distance

$232=2 \mathrm{~mm}$ ), there is little change in osteoblast, chondroblast and fibroblast concentration in callus by increasing bone-plate distance from $2 \mathrm{~mm}$ to $3 \mathrm{~mm}$ (Case 2). However, when the bone-plate distance is increased to $4 \mathrm{~mm}$ (Case 3), the impact of osteoporosis could significantly be alleviated. For example, compared to Case 1, a bone-plate distance of $4 \mathrm{~mm}$ is capable of alleviating the impact of osteoporosis on osteoblasts and chondroblasts concentration by $21 \%$ and $30 \%$, respectively, but there is little change in fibroblast concentration. The implication of these results is that a relatively flexible fixation could potentially encourage endochondral ossification and the development of fracture calls in osteoporotic condition which is favourable for fracture healing [35].

\section{DISCUSSION}

The current model predictions are consistent with the growing evidence that indicates, at early stage of healing, the loading regime required for osteoporotic bone cell simulation is different from that in normal condition $[36,16]$. It generally agrees that the mechanical stimuli mediated bone fracture healing is governed by the movement (IFM) of fracture site $[37,38]$. It is believed that the stiffness of an osteoporotic fracture fixation construct has to be increased as osteoporosis decreases the mechanical properties of bone. However, an overly stiff fixation construct may lead to insufficient IFM at the fracture site which further inhibits the mechanical stimuli mediated healing in osteoporotic condition, resulting ultimately in delayed healing or non-union [39]. While IFM of the fracture site depends on the physiological activities of a patient after surgery, excessive loading could result in complications (e.g. increasing the risk of failure of implant fixation) due to the reduced 
mechanical quality of osteoporotic bone. The present study demonstrates that the application of locking compression plate (LCP) system provides a way of enhancing IFM without increasing the magnitude of the loading (Fig 3) due to the fact that clinically the flexibility of LCP could be enhanced by adjusting the working length, bone-plate and number of screws [40]. The implication of this research is that fixation design can be strategically modified based on the predictions from theoretical models to achieve improved fracture healing outcomes in osteoporotic patients.

At early stage of healing, it is reasonable to assume that the majority of MSCs in callus is from the periosteum, surrounding soft tissues and bone marrow [25]. Further, the current study mainly focused on investigating the effects of reduced MSCs number in osteoporotic bone and the impaired mechano-regulation of osteoporotic bone cells on bone healing progression. However, the effects of osteoporosis on other biological factors that influence the healing outcomes were not taken into consideration in this present study. For example, recent studies found that osteoporosis significantly affects the expression of vascular endothelial growth factor (VEGF) [41], which plays an important role in the formation of new vasculature in callus (i.e. angiogenesis), and so delivers necessary oxygen and nutrients for endochondral ossification [42]. The impaired angiogenesis in osteoporotic condition may also lead to delayed healing or non-union, and therefore requires further investigation.

\section{CONCLUSIONS}

In this present study, we developed a computational model to investigate the impact of impairment of MSCs induced by osteoporosis on bone fracture healing and explore the ways of modifying the flexibility of LCP to improve the healing outcomes in osteoporotic bone. A summary of the main results are as follows:

- The osteoporosis induced decrease in number of MSCs in osteoporotic bone and the 
impaired response of MSCs to their mechanical stimuli could significantly reduce MSCs differentiation to osteoblast and chondroblast at early of healing (e.g. a 50\% reduction factor may lead to $55 \%$ and $47 \%$ decrease in osteoblast and chondroblast concentration in early stage soft callus, respectively).

- $\quad$ By using a more flexible LCP through increasing bone-plate distance, the effects of osteoporosis on healing could be significantly alleviated. For example, by increasing the bone-plate distance from $2 \mathrm{~mm}$ to $4 \mathrm{~mm}$, the comparison of the simulation results from Case 1 and Case 3 shows that the serious impact of osteoporosis on osteoblast and chondroblast concentration in callus can be reduced by $21 \%$ and $30 \%$, respectively.

\section{ACKNOWLEDGEMENTS}

The authors would like to thank AOTRAUMA Asia Pacific (AOTAP14-02) and Victorian Orthopaedic Research Trust (2015-2016) for their supports.

\section{CONFLICT OF INTEREST}

The authors declare that they have no conflict of interest.

\section{REFERENCES}

1. Harvey N, Dennison E, Cooper C (2010) Osteoporosis: impact on health and economics. Nature Reviews Rheumatology 6:99-105

2. Hollevoet N, Verdonk R, Kaufman J-M, Goemaere S (2011) Osteoporotic fracture treatment. Acta Orthop Belg 77 (4):441-447

3. Nikolaou VS, Efstathopoulos N, Kontakis G, Kanakaris NK, Giannoudis PV (2009) The influence of osteoporosis in femoral fracture healing time. Injury 40:663-668

4. Namkung-Matthai H, Appleyard R, Jansen J, Lin JH, Maastricht S, Swain M, Mason RS, Murrell GAC, Diwan AD, Diamond T (2001) Osteoporosis influences the early period of fracture healing in a rat osteoporotic model. Bone 28 (1):80-86

5. Stolzing A, Scutt A (2006) Age-related impairment of mesenchymal progenitor cell function. Aging Cell 5 (3):213-224

6. Marsell R, Einhorn TA (2011) The biology of fracture healing. Injury 42 (6):551-555

7. Quarto R, Thomas D, Liang CT (1995) Bone progenitor cell deficits and the age-associated decline in bone repair capacity. Calcified Tissue International 56 (2):123-129

8. Prall WC, Haasters F, Heggebö J, Polzer H, Schwarz C, Gassner C, Grote S, Anz D, Jäger M, Mutschler W, Schieker M (2013) Mesenchymal stem cells from osteoporotic patients 
feature impaired signal transduction but sustained osteoinduction in response to BMP-2 stimulation. Biochemical and Biophysical Research Communications 440 (4):617-622 9. Haasters F, Docheva D, Gassner C, Popov C, Böcker W, Mutschler W, Schieker M, Prall WC (2014) Mesenchymal stem cells from osteoporotic patients reveal reduced migration and invasion upon stimulation with BMP-2 or BMP-7. Biochemical and Biophysical Research Communications 452 (1):118-123

10. Epari D, Duda G, Thompson M (2010) Mechanobiology of bone healing and regeneration: in vivo models. Proceedings of the Institution of Mechanical Engineers, Part H: Journal of Engineering in Medicine 224 (12):1543-1553

11. Lacroix D, Prendergast PJ, Li G, Marsh D (2002) Biomechanical model to simulate tissue differentiation and bone regeneration: Application to fracture healing. Med Biol Eng Comput 40 (1):14-21

12. Claes LE, Heigele CA, Neidlinger-Wilke C, Kaspar D, Seidl W, Margevicius KJ, Augat P (1998) Effects of mechanical factors on the fracture healing process. Clin Orthop Relat Res 355:S132-S147

13. Zhang L, Richardson M, Mendis P (2012) The role of chemical and mechanical stimuli in mediating bone fracture healing. Clinical and Experimental Pharmacology and Physiology 39:706-710

14. Klein P, Schell H, Streitparth F, Heller M, Kassi J-P, Kandziora F, Bragulla H, Haas NP, Duda GN (2003) The initial phase of fracture healing is specifically sensitive to mechanical conditions. J Orthop Res 21:662-669

15. Lacroix D, Prendergast PJ (2002) A mechano-regulation model for tissue differentiation during fracture healing: analysis of gap size and loading. Journal of Biomechanics 35:11631171

16. Sterck JGH, Klein-Nulend J, Lips P, Burger EH (1998) Response of normal and osteoporotic human bone cells to mechanical stress in vitro. American Journal of Physiology - Endocrinology and Metabolism 274:E1113-E1120

17. Bergman RJ, Gazit D, Kahn AJ, Gruber H, Mcdougall S, Hahn TJ (1996) Age-related changes in osteogenic stem cells in mice. Journal of Bone and Mineral Research 11 (5):568577

18. Giannoudis P, Schneider E (2006) Principles of fixation of osteoporotic fractures. Journal of Bone \& Joint Surgery, British Volume 88 (10):1272-1278

19. Miramini S, Zhang L, Richardson M, Mendis P (2014) Computational simulation of mechanical microenvironment of early stage of bone healing under locking compression plate with dynamic locking screws. Applied Mechanics and Materials 53:281-286

20. Miramini S, Zhang L, Richardson M, Mendis P, Ebeling P (2016) Influence of fracture geometry on bone healing under locking plate fixations: A comparison between oblique and transverse tibial fractures. Medical Engineering and Physics 38 (10):1100-1108

21. Miramini S, Zhang L, Richardson M, Mendis P, Oloyede A, Ebeling P (2016) The relationship between interfragmentary movement and cell differentiation in early fracture healing under locking plate fixation. Australasian Physical \& Engineering Sciences in Medicine 39 (1):123-133

22. Miramini S, Zhang L, Richardson M, Pirpiris M, Mendis P, Oloyede K, Edwards G (2015) Computational simulation of the early stage of bone healing under different Locking Compression Plate configurations. Computer Methods in Biomechanics and Biomedical Engineering 18 (8):900-913

23. Zhang L, Miramini S, Richardson M, Pirpiris M, Mendis P, Oloyede K (2013) The effects of flexible fixation on early stage bone fracture healing. International Journal of Aerospace and Lightweight Structures 3 (2):181-189 
24. Zhang L, Miramini S, Richardson M, Ebeling P, Little D, Yang Y, Huang Z (2017) Computational modelling of bone fracture healing under partial weight-bearing exercise. Med Eng Phys 42:65-72

25. Peiffer V, Gerisch A, Vandepitte D, Oosterwyck HV, Geris L (2011) A hybrid bioregulatory model of angiogenesis during bone fracture healing. Biomechanics and Modeling in Mechanobiology 10 (2):383-395

26. Simpson AHRW, Mills L, Noble B (2006) The role of growth factors and related agents in accelerating fracture healing. Journal of Bone and Joint Surgery 88 (6):701-705

27. Miramini S, Smith DW, Zhang L, Gardiner BS (2017) The spatio-temporal mechanical environment of healthy and injured human cartilage during sustained activity and its role in cartilage damage. Journal of the Mechanical Behavior of Biomedical Materials

28. Perren S (1979) Physical and biological aspects of fracture healing with special reference to internal fixation. Clin Orthop Relat Res 138:175-196

29. Nagel T, Kelly DJ (2010) Mechano-regulation of mesenchymal stem cell differentiation and collagen organisation during skeletal tissue repair. Biomechanics and Modeling in Mechanobiology 9 (3):359-372

30. Andreykiv A, Van Keulen F, Prendergast P (2008) Simulation of fracture healing incorporating mechanoregulation of tissue differentiation and dispersal/proliferation of cells. Biomechanics and modeling in mechanobiology 7 (6):443-461

31. Zhang L, Miramini S, Gardiner BS, Smith DW, Grodzinsky AJ (2015) Time evolution of deformation in a human cartilage under cyclic loading. Ann Biomed Eng 43 (5):1166-1177

32. Huiskes R, Van Driel W, Prendergast P, Søballe K (1997) A biomechanical regulatory model for periprosthetic fibrous-tissue differentiation. J Mater Sci Mater Med 8 (12):785-788 33. González-Torres LA, Gómez-Benito MJ, Doblaré M, García-Aznar JM (2010) Influence of the frequency of the external mechanical stimulus on bone healing: A computational study. Med Eng Phys 32:363-371

34. COMSOL Multiphysics (2012). 4.3 edn. COMSOL Inc,

35. Claes L, Reusch M, Göckelmann M, Ohnmacht M, Wehner T, Amling M, Beil FT, Ignatius A (2011) Metaphyseal fracture healing follows similar biomechanical rules as diaphyseal healing. J Orthop Res 29 (3):425-432

36. Neidlinger-Wilke C, Iris Stalla* LC, Brand R, Hoellen I, Rübenacker S, Arand M, Kinzl L (1995) Human osteoblasts from younger normal and osteoporotic donors show differences in proliferation and TGF- $\beta$ release in response to cyclic strain. Journal of Biomechanics 28 (12): 1411-1141

37. García-Aznar JM, Kuiper JH, Gómez-Benito MJ, Doblaré M, Richardson JB (2007) Computational simulation of fracture healing: Influence of interfragmentary movement on the callus growth. Journal of Biomechanics 40 (7):1467-1476

38. Cheal EJ, Mansmann KA, III AMD, Hayes WC, Perren SM (1991) Role of interfragmentary strain in fracture healing: Ovine model of a healing osteotomy. Journal of Orthopaedic Research 9 (1):131-142

39. Gardner MJ, Nork SE, Huber P, Krieg JC (2010) Less rigid stable fracture fixation in osteoporotic bone using locked plates with near cortical slots. Injury 41 (6):652-656

40. Claes L (2011) Biomechanical principles and mechanobiologic aspects of flexible and locked plating. J Orthop Trauma 25:S4-S7

41. Ding W-G, Wei Z-X, Liu J-B (2010) Reduced local blood supply to the tibial metaphysis is associated with ovariectomy-induced osteoporosis in mice. Connective Tissue Research 52 (1):25-29

42. Beamer B, Hettrich C, Lan J (2010) Vascular endothelial growth factor: an essential component of angiogenesis and fracture healing. HSSJ 6:85-94 
406 43. McCartney W, Donald BM, Hashmi M (2005) Comparative performance of a flexible 407 fixation implant to a rigid implant in static and repetitive incremental loading. Journal of 408 materials processing technology 169 (3):476-484

409 44. Anderson CB (1967) Mechanics of fluids. In: Baumeister, T. (Ed.), Marks' Saturated 410 Handbook of Mechanical Engineers.3.48-43.76

411 45. Schaffler MB, Burr DB (1988) Stiffness of compact bone: effects of porosity and density.

412 J Biomech 21 (1):13-16

413 46. Cowin SC (1999) Bone poroelasticity. J Biomech 32 (3):217-238

414 47. Stoffel K, Dieter U, Stachowiak G, Gächter A, Kuster MS (2003) Biomechanical testing of the LCP-how can stability in locked internal fixators be controlled? Injury 34:11-19

418 Table 1 Parameters used throughout this study [43,11,44-47,25]. 


\begin{tabular}{|c|c|c|c|c|}
\hline \multicolumn{5}{|c|}{ Parametric study } \\
\hline Control & \multicolumn{4}{|c|}{$\begin{array}{l}\mathrm{BPD}=2 \mathrm{~mm}, k_{d}^{s}=k_{d}^{m}=1.0 \\
\mathrm{BPD} \text { - LCP Bone-plate distance; } \\
k_{d}^{s} \text { - Reduction factor of the sensitivity of MSCs to mechanical stimuli; } \\
k_{d}^{m} \text { - Reduction factor of MSC concentration at boundary }\end{array}$} \\
\hline Case 1 & \multicolumn{4}{|c|}{$\mathrm{BPD}=2 \mathrm{~mm}, k_{d}^{s}=k_{d}^{m}=0.5$} \\
\hline Case 2 & \multicolumn{4}{|l|}{$\mathrm{BPD}=3 \mathrm{~mm}, k_{d}^{s}=k_{d}^{m}=0.5$} \\
\hline Case 3 & \multicolumn{4}{|c|}{$\mathrm{BPD}=4 \mathrm{~mm}, k_{d}^{s}=k_{d}^{m}=0.5$} \\
\hline \multicolumn{5}{|c|}{$\begin{array}{l}\text { Material properties of bone } \\
\end{array}$} \\
\hline & & Porosity & Poisson's Ratio & $\begin{array}{l}\text { Permeability } \\
\left(\mathrm{m}^{4} / \mathrm{Ns}\right)\end{array}$ \\
\hline Granulat & Tissue & 0.8 & 0.167 & $10^{-14}$ \\
\hline Marrow & & 0.8 & 0.167 & $10^{-14}$ \\
\hline Cortical & & 0.04 & 0.3 & $10^{-17}$ \\
\hline \multicolumn{5}{|c|}{ Material properties of stainless LCP } \\
\hline \multicolumn{2}{|c|}{ Young's modulus (GPa) } & \multicolumn{3}{|c|}{220} \\
\hline \multicolumn{2}{|c|}{ Poisson's ratio } & \multicolumn{3}{|c|}{0.34} \\
\hline \multicolumn{5}{|c|}{ Material properties of fracture callus for Neo-Hookean material model } \\
\hline \multicolumn{2}{|c|}{ Initial shear modulus $\left(\mu_{0}\right)$} & \multicolumn{3}{|c|}{0.021} \\
\hline \multicolumn{2}{|c|}{ Initial bulk modulus $\left(K_{0}\right)$} & \multicolumn{3}{|c|}{0.025} \\
\hline \multicolumn{5}{|c|}{ Mesenchymal stem cells, chondrocytes, osteoblasts and fibroblasts } \\
\hline \multicolumn{2}{|c|}{$\begin{array}{l}\text { Diffusion coefficient of mesenchymal stem } \\
\text { cells }\left(D_{m}\right)\end{array}$} & \multicolumn{3}{|c|}{$9.765 \times 10^{-13} \mathrm{~m}^{2} / \mathrm{s}$} \\
\hline \multicolumn{2}{|c|}{ Diffusion coefficient of chondrocytes $\left(D_{c}\right)$} & \multicolumn{3}{|c|}{$1 \times 10^{-30} \mathrm{~m}^{2} / \mathrm{s}$} \\
\hline \multicolumn{2}{|c|}{ Diffusion coefficient of osteoblasts $\left(D_{b}\right)$} & \multicolumn{3}{|c|}{$1 \times 10^{-30} \mathrm{~m}^{2} / \mathrm{s}$} \\
\hline \multicolumn{2}{|c|}{ Diffusion coefficient of fibroblasts $\left(D_{f b}\right)$} & \multicolumn{3}{|c|}{$2.84 \times 10^{-12} \mathrm{~m}^{2} / \mathrm{s}$} \\
\hline \multicolumn{2}{|c|}{$\begin{array}{l}\text { Rate of mechanical stimuli mediated } \\
\text { chondrocyte differentiation }\left(s_{d}^{c}\right)\end{array}$} & \multicolumn{3}{|c|}{$0.3 /$ day } \\
\hline \multicolumn{2}{|c|}{$\begin{array}{l}\text { Rate of mechanical stimuli mediated } \\
\text { osteoblast differentiation }\left(s_{d}^{b}\right)\end{array}$} & \multicolumn{3}{|c|}{$\begin{array}{l}(0.005+0.145 \times S) / \text { day, where } S \text { is mechanical } \\
\text { simulation index }\end{array}$} \\
\hline \multicolumn{2}{|c|}{$\begin{array}{l}\text { Rate of mechanical stimuli mediated } \\
\text { fibroblast differentiation }\left(s_{d}^{f b}\right)\end{array}$} & \multicolumn{3}{|c|}{$0.01 /$ day } \\
\hline
\end{tabular}

\section{Figure Caption}

421 Fig 1 The proposed modelling scheme. (a) Scope of this study incorporating impact of osteoporosis on healing outcomes; (b) A schematic diagram of a fractured tibia bone supported by a locking compression plate (LCP) system undergoing physiological relevant 
424 loading. The flexibility of LCP can be adjusted by working length (WL) and bone-plate 425 distance (BPD) of the LCP.

(a)

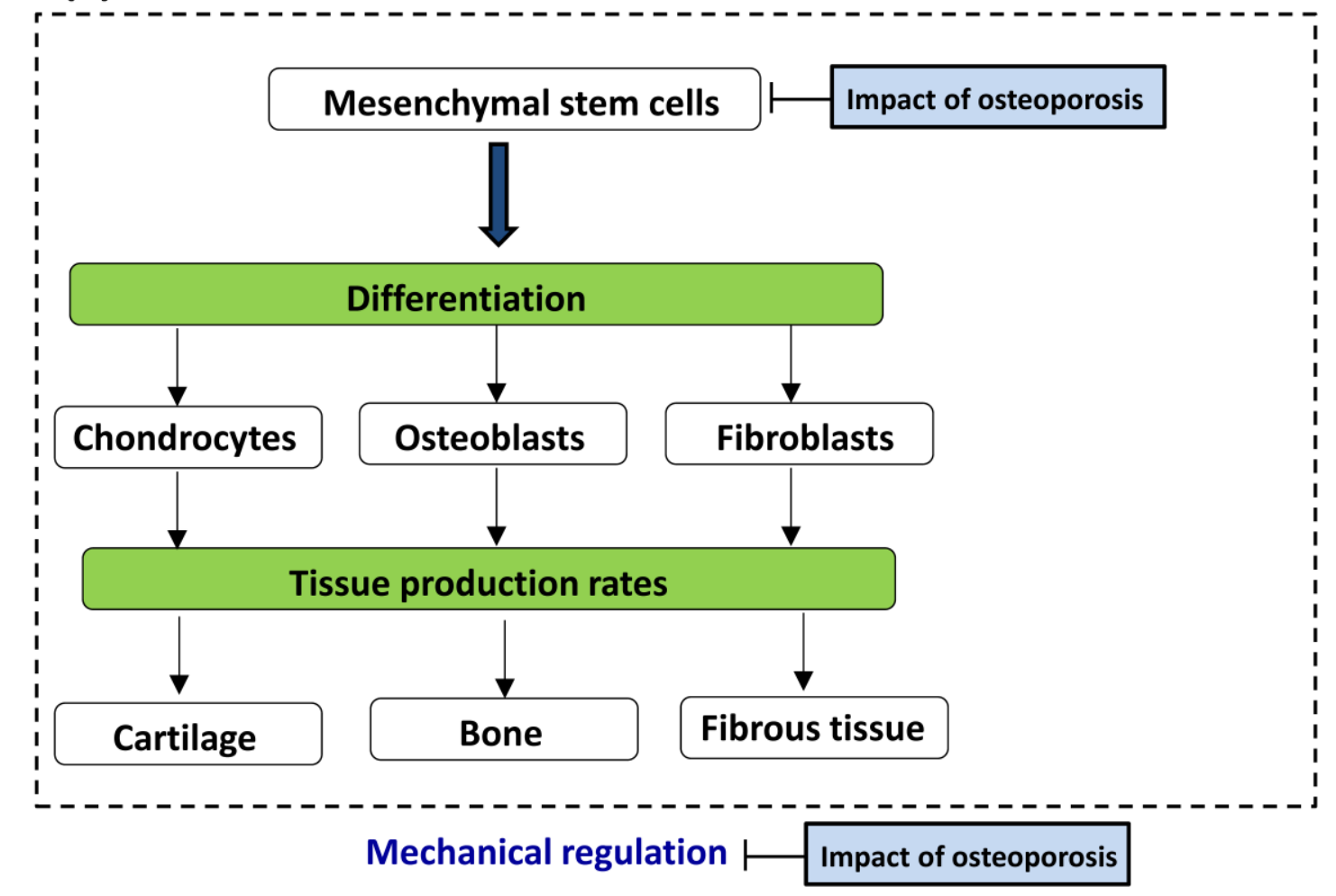



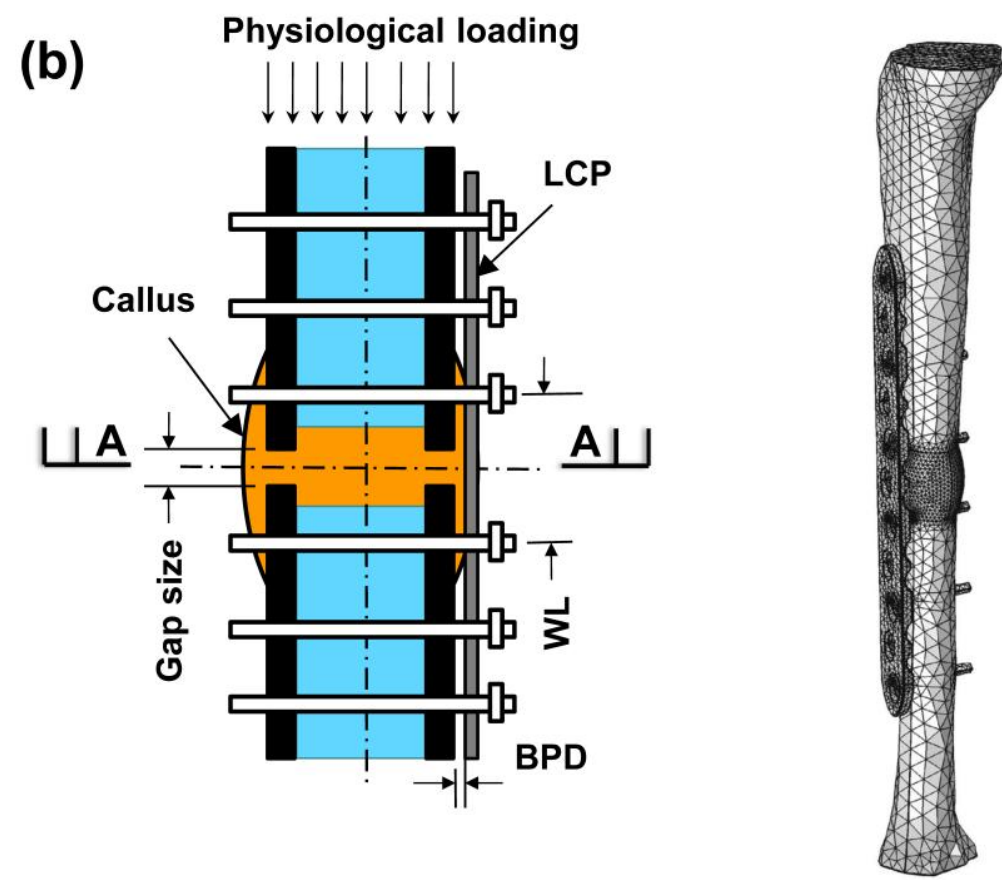

Fig 2 The distribution of reduced mechanical simulation index $(S)$ in the fracture callus 429 (Section A-A) with consideration of the impact of osteoporosis. 


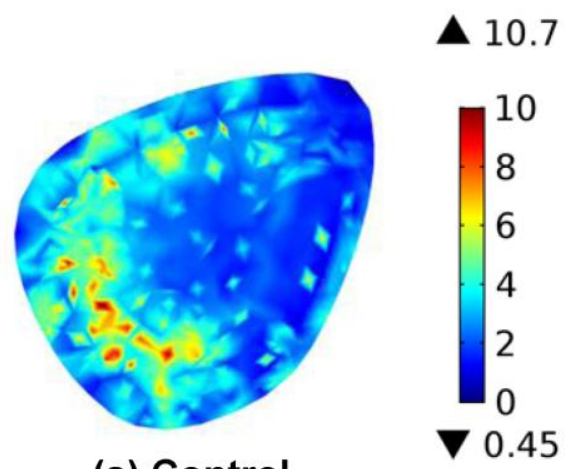

(a) Control

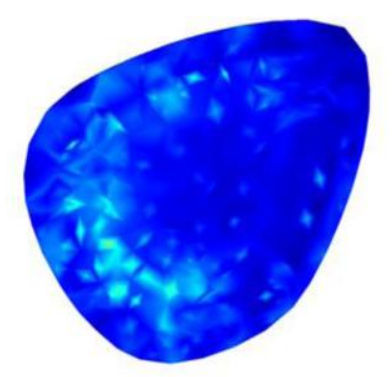

(b) Case 1
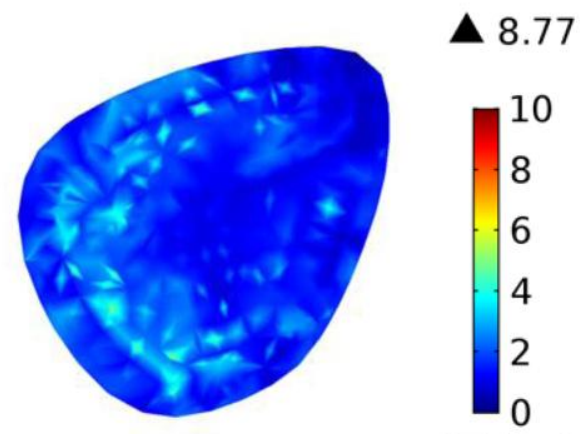

(c) Case 2 $\nabla 0.68$

Fig 3 Percent change in differentiation of chondrocytes, osteoblasts and fibroblasts under different cases (i.e. Cases 1-3, Table 1) compared to control respectively.

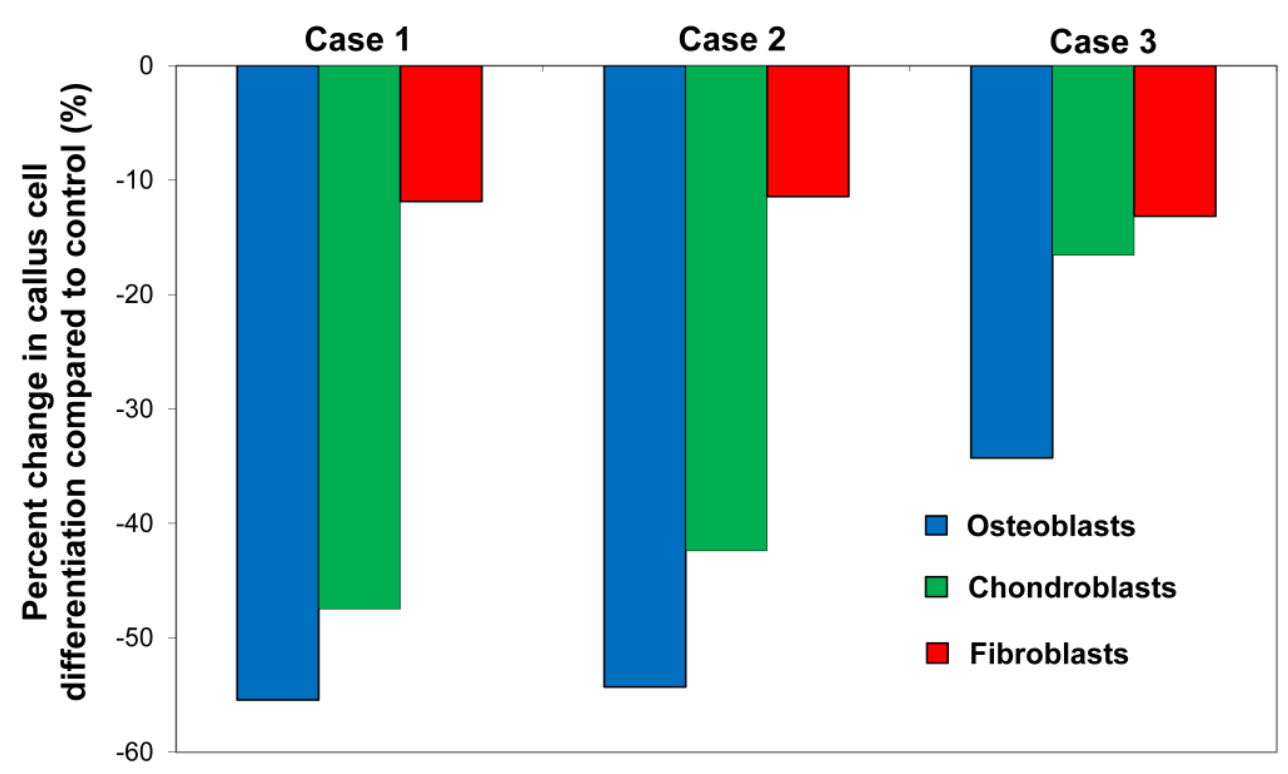

